\title{
In vitro activity of lopinavir/ritonavir and hydroxychloroquine against severe acute respiratory syndrome coronavirus 2 at concentrations achievable by usual doses
}

\author{
Chang Kyung Kang ${ }^{1,}{ }^{\star}$, Moon-Woo Seong ${ }^{2,}{ }^{\star}$, Su-Jin Choi ${ }^{3}$, Taek Soo Kim ${ }^{2}$, Pyoeng Gyun Choe ${ }^{1}$, \\ Sang Hoon Song ${ }^{2}$, Nam-Joong Kim ${ }^{1}$, Wan Beom Park ${ }^{1}$, and Myoung-don Oh ${ }^{1}$
}

Departments of ${ }^{1}$ Internal Medicine and ${ }^{2}$ Laboratory Medicine, Seoul National University College of Medicine, Seoul; ${ }^{3}$ Laboratory of Infection \& Immunity, Seoul National University Hospital Biomedical Research Institute, Seoul, Korea
Received: April 17, 2020

Revised : April 30, 2020

Accepted: May 4, 2020

\section{Correspondence to}

\section{Wan Beom Park, M.D.}

Department of Internal Medi-

cine, Seoul National University

College of Medicine, 101 Dae-

hak-ro, Jongno-gu, Seoul 03080, Korea

Tel: +82-2-2072-3596

Fax: +82-2-762-9662

E-mail: wbpark1@snu.ac.kr https://orcid.org/0000-0003o022-9625

*'These authors contributed equally to this work.
Background/Aims: As the coronavirus disease-2019 global pandemic progresses, screening of antiviral agents effective against severe acute respiratory syndrome coronavirus 2 (SARS-CoV-2) is urgently needed. In addition, considering the viral load kinetics of SARS-CoV-2, which peaks early in the illness, and the massive burden of the disease, which may increase in the near future, identifying well-tolerated oral antivirals becomes increasingly important. We examined the in vitro activity of lopinavir/ritonavir and hydroxychloroquine on SARS$\mathrm{CoV}-2$, at concentrations which can be used to treat coronavirus-19 patients with little concern of toxicity.

Methods: Lopinavir/ritonavir ( $7 / 1.75 \mu \mathrm{g} / \mathrm{mL})$, hydroxychloroquine base (1 or $2 \mu \mathrm{g} /$ $\mathrm{mL}$ ), or a combination thereof were administered 1 hour after the inoculation of SARS-CoV-2 to Vero cells at a multiplicity of infection of 0.05 . We examined cytopathic effects of virus 48 hours after administration of the respective treatments and measured viral loads at three time points (o, 24, and 48 hours posttreatment) by quantitative real-time reverse-transcription polymerase chain reaction, and compared the results obtained from the different antiviral regimens tested.

Results: The severity of cytopathic effects was lower in lopinavir/ritonavir-treated cells, and viral load was significantly reduced in this group compared with the control group $(p<0.001)$. However, hydroxychloroquine did not show significant inhibitory effects on anti-SARS-CoV-2-mediated cytotoxicity or on viral load at either concentration.

Conclusions: Lopinavir/ritonavir showed significant inhibitory effects on SARS-CoV-2 in vitro at its usual plasma concentration. However, the in vitro antiviral activity of hydroxychloroquine at concentrations commonly used in humans was minimal, whether used alone or in combination with lopinavir/ ritonavir.

Keywords: COVID-19; Severe acute respiratory syndrome coronavirus 2; In vitro techniques; Lopinavir/ritonavir; Hydroxychloroquine 


\section{INTRODUCTION}

An outbreak of coronavirus disease-2019 (COVID-19), first reported in Wuhan, China in December of 2019, has become a global health crisis [1]. As of April 29, 2020, a total of 3,018,952 laboratory-confirmed patients have been reported globally, and the number of infected individuals continues to grow.

The disease can cause severe pneumonia and has resulted in 207,973 worldwide fatalities to date and no specific treatment for COVID-19 has yet been established. Therefore, evaluation of therapeutic options for COVID-19 is urgently needed. Moreover, considering the increasingly significant global burden of COVID-19, it is desirable that a potential antiviral agent be safe and orally administered.

Lopinavir/ritonavir, a protease inhibitor that has been safely used to treat human immunodeficiency virus (HIV), showed promise in in vivo and clinical data against severe acute respiratory syndrome coronavirus (SARS-CoV) and Middle East respiratory syndrome coronavirus (MERS-CoV) [2,3]. Its in vitro antiviral activity against severe acute respiratory syndrome coronavirus 2 (SARS-CoV-2), the causative virus of COVID-19, has been recently suggested [4]. Hydroxychloroquine, which has long been used as an antimalarial and immune-modulating drug, has also been proposed as a potent antiviral agent for SARS-CoV [5] and its in vitro activity against SARS-CoV-2 was recently suggested $[6,7]$. However, information focusing on the antiviral activity of hydroxychloroquine at concentrations commonly used in clinical doses is limited.

Therefore, we aimed to explore the in vitro activity of lopinavir/ritonavir, hydroxychloroquine, and their combination against SARS-CoV-2 at concentrations that could be safely administered to SARS-CoV-2 infected patients.

\section{METHODS}

\section{Cells, virus, and antiviral agents}

Chlorocebus aethiops kidney Vero (ATCC CCL-81, ATCC, Manassas, VA, USA) cells were maintained in minimum Eagle's medium (Gibco Invitrogen, Grand Island, NY, USA) supplemented with 10\% fetal bovine serum (Gibco Invitrogen) at $37^{\circ} \mathrm{C}$ in a humidified atmosphere of $5 \% \mathrm{CO}_{2}$.
A clinical isolate of SARS-CoV-2 (BetaCoV/Korea/ SNUo1/2020) was used in this study [8]. All infection experiments were performed in a biosafety level-3 laboratory. Lopinavir/ritonavir (KALETRA, AbbVie, North Chicago, IL, USA) and hydroxychloroquine sulfate (Oxiklorin, Elyson Pharmaceutical Co. Ltd., Seoul, Korea) were dissolved in $99 \%$ ethanol and phosphate-buffered saline, respectively, to prepare stock solutions $[9,10]$. The drugs were titrated to simulate their steady-state plasma concentrations under usual doses; $7 \mathrm{\mu g} / \mathrm{mL}$ of lopinavir combined with $1.75 \mu \mathrm{g} / \mathrm{mL}$ of ritonavir (usual dose of $400 / 100$ mg twice daily for lopinavir/ritonavir) and either 1 or $2 \mu \mathrm{g} / \mathrm{mL}$ of hydroxychloroquine base (usual dose of 400 or $800 \mathrm{mg}$ hydroxychloroquine sulfate once daily) $[9,11]$.

\section{Viral inoculation and treatments}

On the day before inoculation, T-25 flasks (ThermoFisher Scientific Inc., Waltham, MA, USA) were seeded with Vero cells to ensure $80 \%$ confluence on the day of inoculation. On the day of inoculation, the media was removed from the flasks and the cells were infected with SARS-CoV-2 at a multiplicity of infection of 0.05 in $1 \mathrm{~mL}$ of complete media. A control sample was also prepared using media only. All samples were incubated at $37^{\circ} \mathrm{C}$, $5 \% \mathrm{CO}_{2}$, and gently shaken every 10 minutes for 1 hour. After 1 hour of incubation, $6 \mathrm{~mL}$ of complete media with antiviral agents were added to the flasks and incubated at $37^{\circ} \mathrm{C}$ and $5 \% \mathrm{CO}_{2}$ for 48 hours.

The final concentrations of each antiviral agent were as follows: $7 \mu \mathrm{g} / \mathrm{mL}$ of lopinavir (with $1.75 \mu \mathrm{g} / \mathrm{mL}$ of ritonavir), $1 \mu \mathrm{g} / \mathrm{mL}$ of hydroxychloroquine base, $2 \mu \mathrm{g} / \mathrm{mL}$ of hydroxychloroquine base, $7 \mu \mathrm{g} / \mathrm{mL}$ of lopinavir (with $1.75 \mu \mathrm{g} / \mathrm{mL}$ of ritonavir) plus $1 \mu \mathrm{g} / \mathrm{mL}$ of hydroxychloroquine base, and $7 \mu \mathrm{g} / \mathrm{mL}$ of lopinavir (with $1.75 \mu \mathrm{g} / \mathrm{mL}$ of ritonavir) plus $2 \mu \mathrm{g} / \mathrm{mL}$ of hydroxychloroquine base. All experiments were performed twice and in duplicate.

The Vero cells were observed at 48 hours post-treatment to observe the cytopathic effects, including rounding and detachment of cells.

\section{Measurement of viral RNA by quantitative real-time reverse transcription}

The supernatant from each flask was collected at o, 24, and 48-hour post-treatment and was used for RNA extraction. The supernatant was combined with Trizol LS (Life Technologies, Carlsbad, CA, USA) in a 1:3 ratio and 

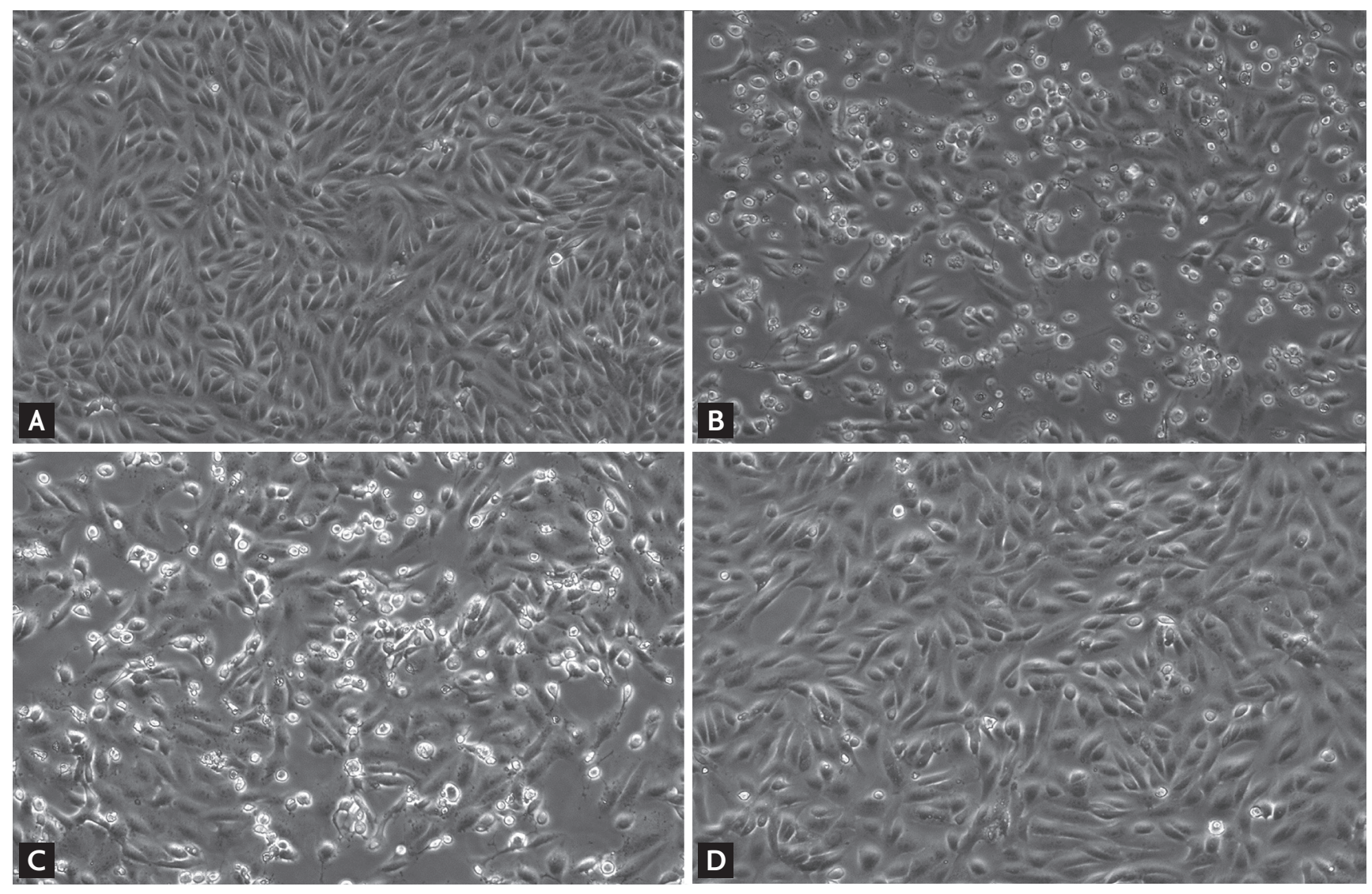

Figure 1. Effect of different antiviral treatments on the cytopathic effects of Vero cells 48 hours after inoculation with severe acute respiratory syndrome coronavirus 2. (A) Control, (B) no antiviral treatment, (C) $2 \mu \mathrm{g} / \mathrm{mL}$ of hydroxychloroquine (expected plasma steady-state concentration of hydroxychloroquine sulfate $800 \mathrm{mg}$ once daily), and (D) 7.0/1.75 $\mu \mathrm{g} / \mathrm{mL}$ of lopinavir/ritona$\operatorname{vir}$ (expected plasma steady-state concentration of lopinavir/ritonavir 400/100 mg twice daily).

incubated for 10 minutes at room temperature to inactivate the virus [12]. RNA was extracted using the MagNA Pure 96 DNA and Viral NA Small volume kit (Roche, Mannheim, Germany) according to the manufacturer's instructions. Viral RNA was detected using the PowerChek 2019-nCoV Real-time polymerase chain reaction (PCR) Kit (Kogene Biotech, Seoul, Korea) for amplification of $\mathrm{E}$ and RdRp genes and quantified using a standard curve which was constructed using in vitro transcribed RNA provided from the European Virus Archive (https://www.european-virus-archive.com).

\section{Statistical analysis}

Viral RNA titers for each treatment were compared using repeated measures analysis of variance and Tukey's multiple comparison test. The change in mean viral loads ac- cording to different treatments was plotted using Prism version 6 (GraphPad Inc., San Diego, CA, USA).

\section{RESULTS}

\section{Changes in cytotoxicity with antiviral treatment}

Fig. 1 and Supplementary Fig. 1 show representative cytopathic effects 48 hours after viral inoculation and administration of antiviral agents. Lopinavir/ritonavir-treated Vero cells showed a significantly lower degree of cytopathic effect, while hydroxychloroquine-treated cells were markedly damaged, at both 1 and $2 \mu \mathrm{g} / \mathrm{mL}$ concentrations. Further, combination treatment did not yield significantly better cell morphology than the lopinavir/ritonavir single treatment. 


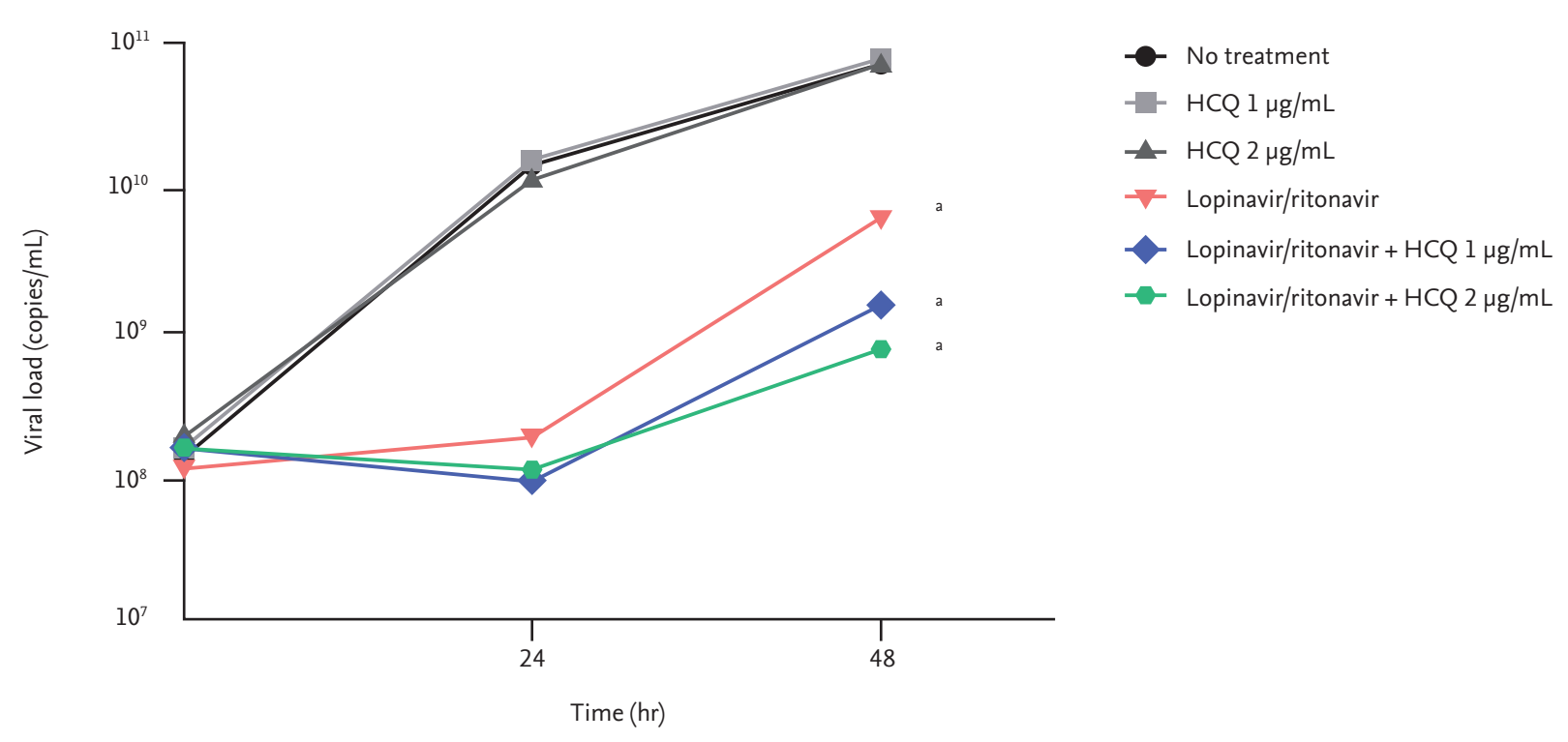

Figure 2. Changes in viral loads in cell culture supernatant in response to different antiviral treatments. Groups are defined as follows: no treatment, hydroxychloroquine (HCQ) base $1 \mu \mathrm{g} / \mathrm{mL}$ (expected plasma steady-state concentration of HCQ sulfate $400 \mathrm{mg}$ once daily), HCQ base $2 \mu \mathrm{g} / \mathrm{mL}$ (expected plasma steady-state concentration of HCQ sulfate 80o mg once daily), lopinavir/ritonavir $7.0 / 1.75 \mu \mathrm{g} / \mathrm{mL}$ (expected plasma steady-state concentration of lopinavir/ritonavir 400/100 mg twice daily), and combinations of lopinavir/ritonavir 7.0/1.75 $\mu \mathrm{g} / \mathrm{mL}$ with HCQ base 1 or $2 \mu \mathrm{g} / \mathrm{mL}$. ${ }^{\mathrm{a}}$ Denotes $p<0.001$ when compared with no treatment group.

\section{Changes in viral RNA with antiviral treatment}

Viral RNA load was significantly lower in the lopinavir/ ritonavir group than in the no-treatment group or hydroxychloroquine groups (all $p<0.001$ ) (Fig. 2). However, the reduction in viral load by hydroxychloroquine treatment was not significant (hydroxychloroquine $1 \mu \mathrm{g} / \mathrm{mL}$ vs. no treatment, $p=0.341$; hydroxychloroquine $2 \mu \mathrm{g} / \mathrm{mL}$ vs. no treatment, $p=0.894)$.

The quantity of viral RNA was lower in the combination groups than in the single treatment lopinavir/ ritonavir group; however, the difference between them was not significant (lopinavir/ritonavir + hydroxychloroquine $1 \mu \mathrm{g} / \mathrm{mL}$ vs. lopinavir/ritonavir, $p=0.933$; lopinavir/ritonavir + hydroxychloroquine $2 \mu \mathrm{g} / \mathrm{mL}$ vs. lopinavir/ritonavir, $p=0.890$ ).

\section{DISCUSSION}

The COVID-19 pandemic has affected most of the countries in the world, causing more than 200,000 mortalities to date; however, there are no proven treatment options against SARS-CoV-2. Therefore, the screening of poten- tial antivirals to fight COVID-19 is urgently needed and led us to assess the in vitro activity of lopinavir/ritonavir and hydroxychloroquine on SARS-CoV-2 at clinically administrable doses. Lopinavir/ritonavir showed marked in vitro activity against SARS-CoV-2 in terms of preventing cytotoxicity and reducing viral load, while hydroxychloroquine did not. The results of this in vitro study could provide insights into the establishment of effective COVID-19 therapeutics.

When considering the viral load kinetics of SARSCoV-2 in the respiratory tract, which peaks early in the illness $[13,14]$, early initiation of treatment might be the key to improve clinical outcomes. Considering the massive burden of the disease, oral antivirals have many advantages over intravenous drugs. We examined the in vitro activity of the oral antivirals lopinavir/ritonavir and hydroxychloroquine against SARS-CoV-2 at their patient administrable doses. These drugs can be readily applied to patients at the levels we studied with little concern of toxicity. Consequently, the results of this study underscore the potential importance of lopinavir/ritonavir treatment against COVID-19.

Lopinavir/ritonavir is a protease inhibitor commonly 
used in the treatment of HIV that interestingly has also been shown to have an antiviral effect on SARS-CoV and MERS-CoV [2,3]. Chu et al. [3] reported the clinical efficacy of lopinavir/ritonavir in SARS-CoV-infected patients by comparing 41 and 111 patients who had been treated with lopinavir/ritonavir plus ribavirin or ribavirin alone. The adverse clinical outcome (acute respiratory distress syndrome or death) was significantly lower in the lopinavir/ ritonavir treated group (2.4\% vs. $28.8 \%, p<0.001$ ) [3]. Similarly, Chan et al. [2] reported significantly better clinical, radiological, and pathological outcomes in the lopinavir/ ritonavir-treated group than in the untreated group in the marmoset-MERS model.

A recent randomized controlled trial involving severe COVID-19 patients revealed no particular benefit of lopinavir/ritonavir administration compared with the standard care [15]. However, the efficacy of lopinavir/ ritonavir may not have been properly evaluated in this study because it was unblinded in nature and the participation of patients was delayed (13 median days [interquartile range, 11 to 16] from disease onset to randomized treatment assignment). Thus, further clinical trials are warranted to explore the efficacy of lopinavir/ritonavir on COVID-19.

Despite several possible adverse reactions, including diarrhea, nausea, abdominal pain, and headache, lopinavir/ritonavir is generally a well-tolerated drug [16]. Considering its promising anti-coronaviral activity, lopinavir/ritonavir is one of the leading safe candidates for the treatment of COVID-19. Our results clearly indicate that lopinavir/ritonavir hold potential as an anti-SARS-CoV-2 agent and should be considered for treating COVID-19 patients in this epidemic.

Two studies have recently reported on the in vitro activity of hydroxychloroquine against SARS-CoV-2 $[6,7]$. Interestingly, the effective concentration $50\left(\mathrm{EC}_{50}\right)$ values of hydroxychloroquine to SARS-CoV-2 varied largely in these studies (ranging from 0.72 to $17.31 \mu \mathrm{M}$ at 48 hours post-treatment), which may be due to the differences in the analyzed drug, inoculum size of the virus, or viral strain. Although in vitro activity of hydroxychloroquine against SARS-CoV-2 in this study tended to be lower than that from one of previous studies [6], it raises a concern on the efficacy of hydroxychloroquine in COVID-19. Furthermore, the combination of hydroxychloroquine and lopinavir/ritonavir did not show a synergistic inhibitory effect on viral load compared to lopinavir/ritonavir treatment alone, implying that usual doses of hydroxychloroquine might not be helpful in the treatment of COVID-19.

There are also ongoing controversies regarding the clinical efficacy of hydroxychloroquine in the treatment ofCOVID-19. Although Gautret et al. [17,18] suggested that hydroxychloroquine with azithromycin could cause rapid decline of viral loads, another small, randomized clinical trial did not reveal a significant reduction in viral load [19]. Additional potential safety issues, such as arrhythmia or sudden death $[20,21]$ need to be considered. Therefore, further evaluations are warranted to determine the clinical utility of hydroxychloroquine against COVID-19.

This study had some limitations. First, we could not examine the in vitro activity of lopinavir/ritonavir and hydroxychloroquine against SARS-CoV-2 at their estimated concentrations from the respiratory tract, owing to the scarcity of information on tissue levels [22]. Second, we did not evaluate $\mathrm{EC}_{50}$ values or pre-attachment activity of the drugs against SARS-CoV-2 in this study because the objective of the present study was to determine their in vitro activity at their clinical doses on an established infection. Finally, we examined the in vitro antiviral activity of lopinavir/ritonavir and hydroxychloroquine against only one SARS-CoV-2 isolate, which was imported from China [8].

In conclusion, this in vitro experimental study showed that lopinavir/ritonavir, at its clinically relevant concentration, showed significant anti-SARS-CoV-2 activity when it was administered following viral infection. Further, our data suggests that the in vitro antiviral activity of hydroxychloroquine at clinically relevant concentrations is minimal, whether used alone or in combination with lopinavir/ritonavir.

\section{KEY MESSAGE}

1. Lopinavir/ritonavir showed significant anti-severe acute respiratory syndrome coronavirus 2 (SARSCoV-2) activity both in terms of the prevention of cytotoxicity and reducing the viral load at plasma concentrations achievable by usual doses.

2. However, hydroxychloroquine did not show significant anti-SARS-CoV-2 activity at plasma concentrations achievable by usual doses. 


\section{Conflict of interest}

No potential conflict of interest relevant to this article was reported.

\section{REFERENCES}

1. World Health Organization. Novel coronavirus (2019$\mathrm{nCoV})$ situation reports [Internet]. Geneva (CH): WHO, 2020 [cited 2020 May 21]. Available from: https://www.who. int/emergencies/diseases/novel-coronavirus-2019/situation-reports/.

2. Chan JF, Yao Y, Yeung ML, et al. Treatment with lopinavir/ ritonavir or interferon- $\beta 1 \mathrm{~b}$ improves outcome of MERS$\mathrm{CoV}$ infection in a nonhuman primate model of common marmoset. J Infect Dis 2015;212:1904-1913.

3. Chu CM, Cheng VC, Hung IF, et al. Role of lopinavir/ ritonavir in the treatment of SARS: initial virological and clinical findings. Thorax 2004;59:252-256.

4. Choy KT, Wong AY, Kaewpreedee P, et al. Remdesivir, lopinavir, emetine, and homoharringtonine inhibit SARSCoV-2 replication in vitro. Antiviral Res 2020;178:104786.

5. Vincent MJ, Bergeron E, Benjannet $S$, et al. Chloroquine is a potent inhibitor of SARS coronavirus infection and spread. Virol J 2005;2:69.

6. Yao X, Ye F, Zhang M, et al. In vitro antiviral activity and projection of optimized dosing design of hydroxychloroquine for the treatment of severe acute respiratory syndrome coronavirus 2 (SARS-CoV-2). Clin Infect Dis 2020 Mar 9 [Epub]. https://doi.org/10.1093/cid/ciaa237.

7. Liu J, Cao R, Xu M, et al. Hydroxychloroquine, a less toxic derivative of chloroquine, is effective in inhibiting SARSCoV-2 infection in vitro. Cell Discov 2020;6:16.

8. Park WB, Kwon NJ, Choi SJ, et al. Virus isolation from the first patient with SARS-CoV-2 in Korea. J Korean Med Sci 2020;35:e84.

9. Food and Drug Administration. Kaletra [Internet]. Silver Spring (MD): FDA, 2017 [cited 2020 May 25]. Available from: https://www.accessdata.fda.gov/drugsatfda_docs/ label/2010/021226so3olbl.pdf.

10. Food and Drug Administration. Plaquenil [Internet]. Silver Spring (MD): FDA, 2017 [cited 2020 May 21]. Available from: https://www.accessdata.fda.gov/drugsatfda_docs/ label/2017/009768s037s045so47lbl.pdf.

11. Jallouli M, Galicier L, Zahr N, et al. Determinants of hydroxychloroquine blood concentration variations in systemic lupus erythematosus. Arthritis Rheumatol 2015;67:2176-2184.

12. Kumar M, Mazur S, Ork BL, et al. Inactivation and safety testing of Middle East respiratory syndrome coronavirus. J Virol Methods 2015;223:13-18.

13. Pan Y, Zhang D, Yang P, Poon LLM, Wang Q. Viral load of SARS-CoV-2 in clinical samples. Lancet Infect Dis 2020;20:411-412.

14. Zou L, Ruan F, Huang M, et al. SARS-CoV-2 viral load in upper respiratory specimens of infected patients. N Engl J Med 2020;382:1177-1179.

15. Cao B, Wang Y, Wen D, et al. A trial of lopinavir-ritonavir in adults hospitalized with severe COVID-19. N Engl J Med 2020;382:1787-1799.

16. Hicks C, King MS, Gulick RM, et al. Long-term safety and durable antiretroviral activity of lopinavir/ritonavir in treatment-naive patients: 4 year follow-up study. AIDS 2004;18:775-779.

17. Gautret P, Lagier JC, Parola P, et al. Clinical and microbiological effect of a combination of hydroxychloroquine and azithromycin in 80 COVID-19 patients with at least a six-day follow up: a pilot observational study. Travel Med Infect Dis 2020;34:101663.

18. Gautret P, Lagier JC, Parola P, et al. Hydroxychloroquine and azithromycin as a treatment of COVID-19: results of an open-label non-randomized clinical trial. Int J Antimicrob Agents 2020 Mar 20 [Epub]. https://doi.org/10.1016/ j.ijantimicag.2020.105949.

19. Chen J, Liu D, Liu L, et al. A pilot study of hydroxychloroquine in treatment of patients with moderate COVID-19. Zhejiang Da Xue Xue Bao Yi Xue Ban 2020;49:215-219.

20. Chatre C, Roubille F, Vernhet H, Jorgensen C, Pers YM. Cardiac complications attributed to chloroquine and hydroxychloroquine: a systematic review of the literature. Drug Saf 2018;41:919-931.

21. Juurlink DN. Safety considerations with chloroquine, hydroxychloroquine and azithromycin in the management of SARS-CoV-2 infection. CMAJ 2020;192:E45O-E453.

22. Arnold SLM, Buckner F. Hydroxychloroquine for treatment of SARS-CoV-2 infection? Improving our confidence in a model-based approach to dose selection. Clin Transl Sci 2020 Apr 8 [Epub]. https://doi.org/10.1111/ cts.12797. 


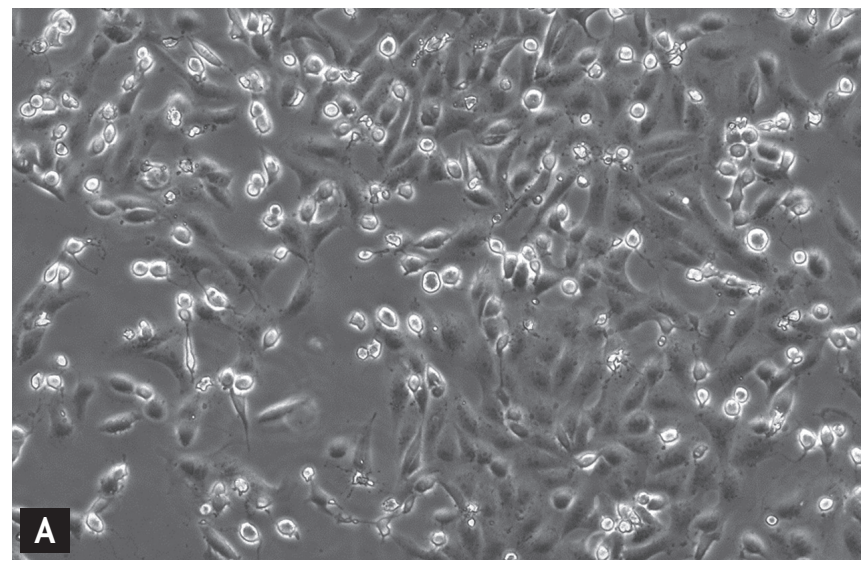

$\mathrm{HCQ}(1 \mu \mathrm{g} / \mathrm{mL})$

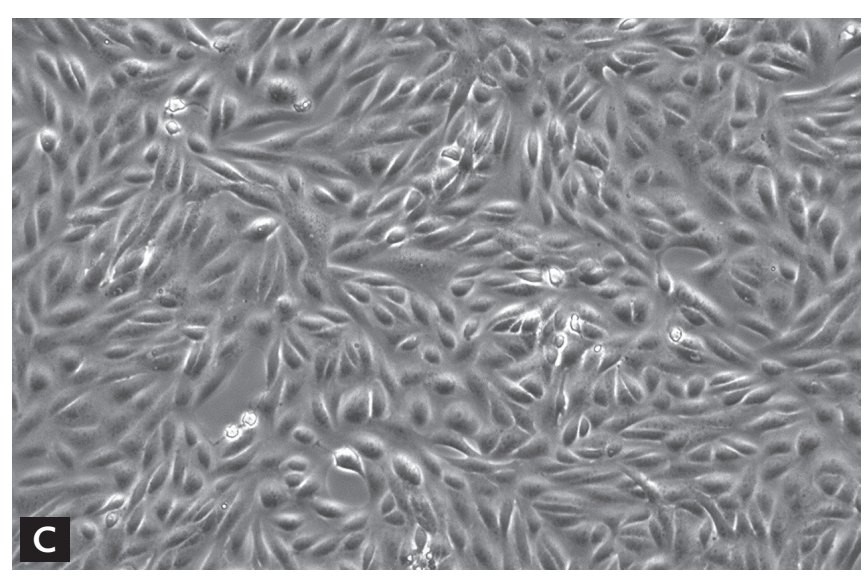

Lopinavir/ritonavir + HCQ (2 $\mu \mathrm{g} / \mathrm{mL})$

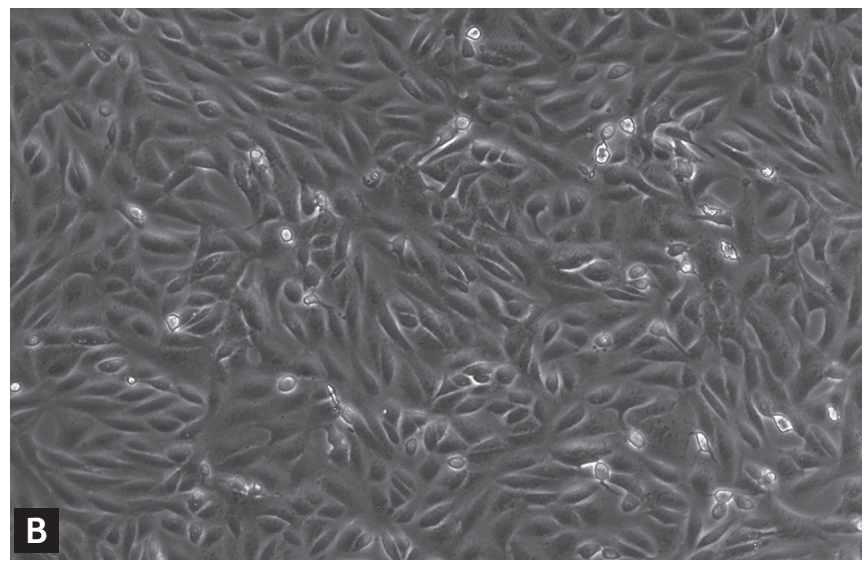

Lopinavir/ritonavir + HCQ (1 $\mu \mathrm{g} / \mathrm{mL})$

Supplementary Figure 1. Representative cytopathic effects of Vero cells after 48 hours of the inoculation of severe acute respiratory syndrome coronavirus 2 according to different antiviral treatment. (A) A $1 \mu \mathrm{g} / \mathrm{mL}$ of hydroxychloroquine (HCQ) base (expected from HCQ sulfate $400 \mathrm{mg}$ daily), (B) 7.0/1.75 $\mu \mathrm{g} / \mathrm{mL}$ of lopinavir/ritonavir (expected from 400/100 $\mathrm{mg}$ twice daily) plus $1 \mu \mathrm{g} / \mathrm{mL}$ of HCQ base, and (C) 7.0/1.75 $\mu \mathrm{g} / \mathrm{mL}$ of lopinavir/ritonavir plus $2 \mu \mathrm{g} / \mathrm{mL}$ of HCQ base (expected from HCQ sulfate $800 \mathrm{mg}$ daily). 